Mots. Les langages du politique

$114 \mid 2017$

Le rapport, entre description et recommandation

\title{
Le rapport : opérativité d'un genre hétérogène
}

The report: operativity of a heterogeneous genre

El informe: operatividad de género heterogéneo

Émilie Née, Claire Oger et Frédérique Sitri

\section{OpenEdition}

\section{Journals}

Édition électronique

URL : https://journals.openedition.org/mots/22752

DOI : $10.4000 /$ mots. 22752

ISSN : 1960-6001

Éditeur

ENS Éditions

Édition imprimée

Date de publication : 10 juillet 2017

Pagination : 9-24

ISSN : 0243-6450

Référence électronique

Émilie Née, Claire Oger et Frédérique Sitri, «Le rapport : opérativité d'un genre hétérogène », Mots. Les langages du politique [En ligne], 114 | 2017, mis en ligne le 10 juillet 2017, consulté le 23 avril 2022 URL : http://journals.openedition.org/mots/22752 ; DOI : https://doi.org/10.4000/mots.22752 


\section{Le rapport : opérativité d’un genre hétérogène}

Dans les institutions et les organisations (appareils gouvernementaux, organisations internationales, entreprises, services sociaux, etc.), la production de rapports est aujourd'hui devenue une pratique professionnelle répandue, réputée fournir un outil privilégié d'aide à la décision. Sans doute peut-on mettre ce développement en relation avec la place croissante de l'évaluation (Bouchard, 2015; Cassin, 2014) et de la production d'indicateurs caractéristique du gouvernement «par les instruments» (Lascoumes et Le Galès, 2004) aussi bien que de la vogue du reporting dans l'entreprise. Dans ce cadre, le rapport peut être envisagé comme un outil de rationalisation et de normalisation de l'action publique comme de l'activité professionnelle : indissociablement descriptif et évaluatif, l'état des lieux qui y est proposé peut être mis au service de préconisations formulées de manière plus ou moins explicite. Publicisé, le rapport est aussi un outil de communication, de promotion, voire de positionnement stratégique.

Par delà la variété des noms employés pour le désigner, et les formes d'organisation textuelle qu'il peut recouvrir - rapport d'information, rapport public, rapport d'activité, rapport de police, rapport éducatif, « rapportage» des États européens auprès de la Commission européenne, etc. - on se propose d'envisager ici le rapport comme un genre ou plutôt un «macro-genre» discursif historiquement situé (Karila-Cohen, 2000 et 2008; Gardey, 2008), ancré dans une sphère sociale d'activité précise, pourvu d'une double visée informative et prescriptive identifiable à des formes langagières récurrentes et s'articulant au sein d'une sphère d'activité donnée avec des genres voisins - par exemple, dans la sphère politico-institutionnelle, les notes, comptes rendus de séances, livres blancs, etc.

Université Paris-Est-Créteil, Céditec

emilie.nee@u-pec.fr

Université Paris-Est-Créteil, Céditec

cla.oger@gmail.com

Université Paris-Ouest-Nanterre, Modyco

fsitri@u-paris1o.fr 
L'ensemble des articles réunis dans ce dossier manifeste la fécondité de ce postulat, qui permet d'envisager les textes à la fois dans leurs conditions de production spécifiques et comme matérialité langagière, d'articuler la description des formes et l'analyse de leurs effets pragmatiques.

\section{Le rapport, un macro-genre}

On s'arrêtera donc dans un premier temps sur cette catégorisation en termes de "genre» : en réfléchissant à une définition opératoire de cette notion, on en montrera du même coup l'intérêt intrinsèque et la portée heuristique pour l'analyse des textes dénommés «rapports».

La notion de genre ne réfère plus aujourd'hui, on le sait, aux seuls genres littéraires ou rhétoriques. Le texte fondateur de Mikhaïl Bakhtine (1984 [19521953], p. 263-308) comme les travaux de Dell Hymes, repris entre autres par Douglas Biber, John Swales ou Charles Bazerman (ou du côté francophone par Jean-Claude Beacco ou Catherine Kerbrat-Orecchioni) ont largement contribué à en étendre l'usage aux productions orales ou écrites les plus diverses, de sorte qu'aujourd'hui nombre de disciplines - et au premier chef l'analyse de discours - en ont fait une notion centrale de leur dispositif théorique et méthodologique. En effet, si la catégorie du genre se révèle utile dans une perspective documentaire de classification des textes, elle permet surtout, et c'est ce qui nous intéresse ici, de rendre compte de la présence et de l'interprétation des formes de langue en relation avec des déterminations diverses, de nature extra-linguistique, qui pèsent sur le discours : notion «biface » (BrancaRosoff, 1999b, p. 116), le genre a pour propriété d'articuler langagier et nonlangagier, ce qui implique d'envisager les productions verbales dans le cadre d'un dispositif énonciatif, en relation avec des pratiques sociales insérées dans une sphère d'activité (Bakhtine), qui en définissent les buts pragmatiques. De façon assez proche, pour Dominique Maingueneau, relayé ici par Rachele Raus, le genre est un « dispositif de communication à la fois social et verbal, historiquement défini » (Maingueneau, 2002).

Les articles réunis dans ce volume décrivent ainsi minutieusement les conditions de production des textes analysés, le cadre institutionnel et le contexte politique ou social plus large dans lequel ils s'insèrent (Parlement européen, Haut Conseil à l'intégration, Gouvernement français par exemple). Mais ils s'intéressent également aux récurrences formelles, les «types relativement stables d'énoncés» dans les termes de Bakhtine, qui constituent le versant «langagier» du genre, et à la façon dont ces récurrences formelles s'articulent avec l'opérativité des textes.

Au-delà de ces considérations relativement générales, le fait de regrouper une série de textes sous une même étiquette générique ne va pas nécessaire- 
ment de soi. De fait on pourrait considérer qu'une telle opération ne consiste pas seulement à identifier des catégories «naturelles» mais est construite - partiellement tout au moins - par le chercheur. Dans le cas qui nous occupe, la catégorisation en genre revêt en elle-même une dimension heuristique, dans la mesure où elle conduit à se demander ce qui justifie de rapprocher les textes portant la dénomination de « rapport». En effet les dénominations que les locuteurs attribuent aux textes - les «noms de genre », si elles constituent un indice de leurs représentations spontanées et de la façon dont ils évaluent ces textes, ne sauraient constituer le seul principe de classement générique / d'identification des genres. Comme le souligne Sonia Branca-Rosoff (1999a, p. 6) :

On trouve [ainsi] des «dissertations» qui n'ont presque aucune des particularités langagières du genre mais qui sont rapportées à une pratique sociale spécifique (un exercice proposé dans le cadre institutionnel de la classe par un professeur); par ailleurs, il arrive qu'on qualifie de «dissertation» des textes sans rapport avec la classe parce que les instruments descriptifs dont nous disposons y reconnaissent des marques linguistiques solidaires et récurrentes dans les dissertations (par exemple, un certain type de plan allié à des procédures de généralisation, à l'usage de la troisième personne et à la quasi absence des modalisations).

C'est pour rendre compte de ce caractère dynamique des genres que JeanMichel Adam et Ute Heidmann proposent la notion de "généricité », qui rend compte du fait qu'un texte est lu différemment selon le genre auquel on le rattache. Cette catégorie est mise en œuvre ici par Mathilde Roussigné pour analyser un corpus de textes littéraires se donnant pour des rapports : "L'approche dynamique selon les degrés de "généricité" des textes plutôt que selon leur appartenance à une catégorie unique (Adam, 2011) permettra de saisir la complexité de leurs fonctionnements et de leurs effets pragmatiques».

Caroline Mellet et Frédérique Sitri (2010) proposent de leur côté de caractériser les genres comme «interaction dynamique » entre nom de genre, visée pragmatique et récurrences formelles. Au vu de la diversité des textes dénommés «rapports», de la variété des sphères dans lesquelles ils sont produits et circulent - et dont rend compte la spécification qui complète le plus souvent le nom «rapport» (d'activités, d’information, de mission, etc.), on considère ici le «rapport» comme un «macro-genre » caractérisé par l'acte de «rapporter» (verbalement) que l'on pourrait caractériser grossièrement de la façon suivante : «dire ce qui est à l'intention d'un destinataire intéressé »1.

1. Il ne serait pas sans intérêt d'approfondir la description sémantique du lexème « rapport » et du verbe « rapporter», en relation avec la présence d'une double suffixation marquant un déplacement, «ad » et « re ». Le déplacement marqué par « re » peut être spatial ou temporel, et s’actualise dans trois valeurs principales : réitération, retour, modification (Vogüé, 1990 et Jalenques, 2002 par exemple). Il est possible que dans le «rapport» au sens où nous l'entendons ici la deuxième valeur soit prépondérante, indiquant dans la définition même du genre une sorte de retour à l'envoyeur. Il serait également intéressant de poursuivre la comparaison entre rapporter / rapport et compte rendu / rendre compte. 
Le caractère «adressé » du rapport pourrait ainsi être considéré comme une dimension constitutive du genre et la description des conditions de production d'un rapport intègre bien souvent la figure du «commanditaire», laquelle peut être distinguée de celle du ou des « destinataires », récepteurs et lecteurs du rapport. La figure du commanditaire est particulièrement importante dans le cas des rapports publics et le double adressage (commanditaire / destinataire) figure par exemple dans les incipit des rapports au Haut Conseil à l'intégration analysés par Naomi Truan et Léa Renard qui montrent que, dans les rapports français, «la mention explicite des commanditaires est une constante», ce qui traduit une «personnalisation plus grande des rapports français». Mathilde Roussigné, étudiant un corpus de textes littéraires qui, se donnant pour des rapports, visent à en démonter les normes de genre, montre comment le texte intitulé La Base fait de cette relation au commanditaire le moteur de l'écriture : ce texte «s'attache surtout à définir une situation d'énonciation unique dans laquelle un rapporteur expose ce que l'on peut nommer sa relation à un commanditaire». Si, comme le souligne M. Bakhtine, tout discours dialogue avec les discours à venir, et en tant que tel, est déterminé par la figure d'un destinataire plus ou moins précis et incarné, on peut dire que dans le cas du rapport, cette figure du destinataire participe à la construction même du texte et peut dès lors donner lieu à des marques repérables. On peut faire l'hypothèse que cette orientation vers le destinataire s'accompagne réciproquement de ce qu'on pourrait appeler «l'engagement» de l'énonciateur dans son texte. En fait, tout se passe comme si dans le rapport «dire ce qui est» - qui ressortit à un simple « compte rendu » - était indissociable d'un « dire comment cela devrait être » répondant à la commande du destinataire et marquant l'engagement de l'énonciateur dans une activité évaluative orientée argumentativement. Tout l'enjeu des rapports sur le microcrédit décrits par Odile Vallée consiste par exemple à accumuler les procédures de validation qui attestent de la réalité des effets positifs du microcrédit : notes de bas de page avec références à un discours d'expert, chiffres, témoignages.

La double visée INFORMER / DÉCRIRE + ÉVALUER / PRESCRIRE qui permettrait de caractériser le macro-genre du «rapport» s'actualise bien entendu, nous l'avons dit, sous des formes extrêmement différentes selon les sphères d'activité, lesquelles définissent l'objet sur lequel porte l'évaluation mais aussi le circuit énonciatif et le dispositif institutionnel dans lequel est pris le rapport. Ainsi un rapport éducatif produit par un service de protection de l'enfance à destination d'un juge pour décrire et caractériser la situation d'un enfant présente-t-il des marques explicites d'un scripteur souvent collectif, dans la mesure où l'enjeu de ces textes est aussi de valoriser l'action éducative et d'en justifier les choix : malgré les consignes d'objectivité et de neutralité diffusées par des guides de rédaction ou les chefs de service, récits, descriptions et analyses sont pris en charge par le scripteur (voir Née, Sitri et Fleury, 2014). Dans la sphère économique, les rapports d'activités des entreprises qui visent à rendre 
compte de la situation de l'entreprise tout en rassurant personnels et actionnaires (voire décideurs publics), comprennent une dimension promotionnelle forte, marquée par l'omniprésence du nom de la marque et d'axiologiques positifs ou de configurations comme la concession. De nombreux rapports sur lesquels porte ce dossier appartiennent quant à eux à la sphère de l'action publique (ministères, grandes organisations internationales, etc.), caractérisée par une forme de «neutralisation discursive » décrite par Claire Oger (2013) ou de dépolitisation (Cussò et Gobin, 2008) qui peut entrer en tension avec la double visée descriptive / prescriptive, la construction d'une figure du destinataire et l'implication de l'énonciateur que nous avons décrites. Le paramètre de la langue peut aussi jouer à ce stade. Ainsi Rachele Raus comparet-elle les versions françaises et italiennes des rapports d'initiative européens concernant la prostitution, rédigés entre 2004 et 2014 par la Commission des droits de la femme et de l'égalité des chances. Elle montre ainsi que «la version française marque davantage la prescription et l'incitation et finit par restituer un dit où l'on arrive parfois à distinguer la présence de voix précises » tandis que N. Truan et L. Renard, analysant des rapports français et allemand sur l'intégration mettent en évidence des «contraintes génériques propres au rapport administratif, qui se défait difficilement de son carcan institutionnel, dont les tentatives de neutralité et d'appel au plus grand nombre, communes aux rapports français et allemands, sont le signe, même si les marqueurs linguistiques diffèrent ». L'apport de la contribution de $M$. Roussigné sur ce point est précisément de montrer comment des textes littéraires qui se donnent les «apparences génériques» du rapport en s’inscrivant dans une "scène générique » (Maingueneau, 2004) autre, celle du poème ou du texte de fiction, « en détournant le rapport de ses contextes de production traditionnels et en mettant à l'épreuve la langue qui le fonde » rendent visibles «les conventions discursives de ces derniers », et en premier lieu les effets liés au suremploi de routines discursives dénuées de sens et de valeur pragmatique.

\section{Hétérogénéité linguistique et sémiotique du rapport}

Un deuxième axe de recherche consiste à étudier dans les textes les manifestations linguistiques et discursives de cette double visée, en les articulant aux déterminations liées à la sphère d'activité dont émane le rapport, à l'objet sur lequel il porte, au dispositif énonciatif dans lequel il est pris... Des observables tels que la composition, les marques énonciatives, les types d'énoncés et les types de textes, ou encore la phraséologie peuvent ainsi être analysés sous cet angle.

Ainsi Émilie Née, Frédérique Sitri et Marie Veniard (2014 et 2016) mettentelles au jour dans des rapports éducatifs émanant de la sphère de l'éducation 
spécialisée et du champ de l'enfance en danger des segments, des agencements lexico-syntaxiques et des motifs récurrents², qu'elles rapportent aux visées descriptives et évaluatives de ces rapports et montrent comment ces deux visées sont, la plupart du temps, fortement intriquées.

Par exemple, des énoncés comme :

Elle est également autonome dans les jeux, et peut rester un long moment à jouer seule dans sa chambre [nous soulignons]

C'est pourquoi aujourd'hui il a évolué dans ses réflexions et peut mesurer un peu plus l'importance d'une telle formation pour son avenir professionnel [nous soulignons]

mettent en jeu, avec le patron récurrent Et peut + inf, une routine argumentative découlant du « rôle assigné à l'éducateur : observer [puis décrire] et évaluer» (Née, Sitri et Veniard, 2016, p. 85). Ce patron récurrent « s’insère [en effet] dans le schéma pragmatique suivant : JUGEMENT D'ÉTAT - CAPACITÉ et / ou ÉVÉNEMENT NOTABLE » et «sa fonction est la preuve par l'exemple» (Ibid.).

Ces routines discursives caractéristiques des rapports éducatifs 3 sont ellesmêmes les composantes d'un type d'enchaînement configurant de manière exemplaire cette double visée, la séquence évaluative (Née, Sitri, Veniard et Fleury, 2017). Cette dernière « est structurée par un mouvement articulant généralisation (analyse et catégorisation) et particularisation (présentation de faits ou de dires) » (Ibid). Le mouvement de particularisation met en jeu des énoncés descriptifs qui sont en relation d'étayage avec des énoncés plus généraux, qui soutiennent un jugement ou une évaluation globale, et qui ont en cela une fonction argumentative - d'exemplification, de justification (voir exemples cidessus).

Une des composantes importantes du rapport est la représentation de discours autres, de dires tenus ailleurs (voir Sitri, 2015). À ce titre, plusieurs contributions de ce numéro explorent un ou plusieurs aspects de la dimension dialogique des rapports4. Quelles que soient les sphères d'où ils émanent, le document désigné du nom de «rapport» peut s’appuyer sur des discours tenus ailleurs et les intégrer au discours qu’il produit, les orientant par là même vers une certaine conclusion. Le genre rapport est donc d'abord dialo-

2. Ces formes sont regroupées par les auteures sous le terme de patrons lexico-syntaxique, qui sont définis comme des «moules syntaxiques ou séquentiels avec une combinatoire lexicale plus ou moins restreinte» (Née, Sitri et Veniard, 2016, p. 77), et sont situées sur « un continuum entre des séquences très figées et d'autres qui le sont moins [...] le moindre degré de figement [étant] représenté par des associations notionnelles» (Ibid.).

3. Pour les auteures, une routine discursive « consiste en la mise en relation de séquences linguistiques récurrentes, partiellement figées (i. e. les patrons définis plus haut) avec des déterminations discursives et des fonctions textuelles propres à un genre ou une sphère d'activité. » (Née, Sitri, Veniard, 2016, p.78; nous soulignons)

4. Lire en particulier les articles d'Andrea Cattelani et Amaia Errecart, de Caroline Ollivier-Yaniv et d’Odile Vallée. 
gique en ce qu'il représente le discours de l'autre à des fins descriptives et évaluatives / argumentatives. Dialogique, le rapport l'est aussi constitutivement dans les parties explicitement argumentatives en ce qu'il marque un positionnement par rapport à un autre discours.

Suivant les sphères, on observe différentes manières de représenter les discours autres et différentes fonctions de ces représentations. Andrea Cattelani et Amaia Errecart étudient ici les rapports dédiés à la « responsabilité sociétale et environnementale des entreprises » (RSE) produits par trois grandes entreprises françaises (Lafarge, BNP Paribas et Total), en se focalisant sur la mise en discours des différentes « parties prenantes » externes de ces entreprises et sur l'exploration des formes verbales et visuelles prises par la figure de l'autre dans ces productions communicationnelles. Les auteurs décrivent alors le caractère plurilogal de ce type de rapport, qui promeut le discours des parties prenantes pour le faire converger vers un discours légitimant l'action de l'entreprise.

Les rapports émanant de la sphère politique et institutionnelle (au niveau national, européen ou international) manifestent quant à eux un positionnement qui constitue un arbitrage implicite entre les prises de positions exprimées dans les débats dont il rend partiellement compte. Le rapport peut dès lors constituer "une arène », comme le décrit ici Caroline Ollivier-Yaniv, à côté d'autres espaces de débats comme les médias ou les réseaux sociaux. L'auteure étudie ainsi un rapport public sur la politique vaccinale, le rapport Hurel, qui est produit dans un contexte de controverse. Confié à une élue, ce document correspond au genre du rapport public dans sa dimension polyphonique et interdiscursive, différentes voix y sont repérables. Ce qui fait la spécificité de ce rapport public, c'est peut-être, comme le souligne l'auteure, le fait d'y faire entrer « des voix non seulement nombreuses [...] mais aussi très rarement rassemblées», correspondant à des acteurs «exceptionnellement diversifiés», dont les positions sont divergentes voire nettement opposées. C. Ollivier-Yaniv propose de parler d' «espace polyphonique inédit» pour ce rapport. Eu égard à l'objet sur lequel il porte, la politique de vaccination en France, ce document instaure ainsi un texte et un discours inédit, assurant la reconnaissance d'une pluralité d'acteurs sur ce problème public, rendant visibles dans le corpus institutionnel certains acteurs qui en sont habituellement absents (les associations de patients ou d'usagers, notamment), «déconfinant » ou publicisant des échanges réservés à certains espaces (réunissant des agences sanitaires, par exemple), produisant enfin un état des lieux conflictuel de la vaccination alors que pour une partie des acteurs institutionnels, politiques, scientifiques légitimes, «la vaccination, ça ne se discute pas».

On peut voir à travers cet exemple comment le genre du rapport participe à la construction d'un problème public, figurant un espace de débat dont, compte tenu du statut de ses acteurs, l'auteure interroge le caractère effectif et durable. 
Un autre type de dynamique à mi-chemin entre le rapport d'entreprise et le rapport public est mis au jour par Odile Vallée, dans son article, qui porte sur douze rapports d'activité produits par la Campagne du Sommet du microcrédit de 1999 à 2012. La visée de ces rapports consiste à «faire reconnaître le microcrédit comme un outil incontournable de la lutte contre la pauvreté ». Les discours d'acteurs hétérogènes sont convoqués, qui défendent des positions différentes voire divergentes sur le microcrédit. Le rapport tient aussi compte des oppositions au microcrédit par des publics tiers et le dialogisme sert ici à «aliment[er] la dimension politique du rapport». Les voix de l'autre sont néanmoins orchestrées et agencées de manière à proposer un discours cohérent sur le microcrédit, à conforter le point de vue défendu par le discours du rapport et à consolider la position tenue par la campagne de microcrédit au sein d'une pluralité d'acteurs : celle-ci «doit [ainsi] organiser la polyphonie (Todorov, 1981) pour réinventer au fil du temps la cohérence globale de son discours et, ainsi, légitimer son statut de tiers référent et animateur du secteur de la microfinance».

Dans les rapports émanant de la sphère institutionnelle, les différents procédés de neutralisation et de «dépolitisation » déjà évoqués se doublent d'un lissage énonciatif qui atténue ou passe sous silence certaines positions jugées excessives. Dans l'article de C. Ollivier-Yaniv, le positionnement de la rapporteure joue à ce titre un rôle important : les dénonciations du rôle des laboratoires pharmaceutiques, par exemple, ne sont pas même évoquées, «considérées par la députée responsable du rapport comme apparentées à une théorie du complot». De même, l'article de R. Raus s'attache à montrer comment la visée incitative du discours du rapport procède d' "un aplatissement de la dimension argumentative ». Ce que dévoile la comparaison de traductions, c'est, outre l'étagement énonciatif lié au processus même de traduction, la différenciation des phénomènes de neutralisation. Dans le rapport traduit en italien «le dit reste polémique, ouvert aux autres en raison sans doute d'un imaginaire ressentant le positionnement italien comme moins légitime. Par contre, l'énonciateur-traducteur français tend à mettre en valeur ses propres idées et à proposer ses solutions, sentant son positionnement comme légitime et autoritaire».

Enfin, une des composantes du rapport qui se trouve abordée par plusieurs des articles et qui manifeste toujours la double visée des rapports est l'hétérogénéité sémiotique et textuelle, qui se matérialise dans la place et l'usage des chiffres, graphiques, illustrations (Bouchard, 2008; Bacot, Desmarchelier et Rémi-Giraud, 2012) et dans la diversité des genres et types textuels qui structurent les rapports (portraits, témoignages, listes, description, argumentation, narration, etc.). Cette composante nécessite des approches sémio-discursives, qui s'appuient, dans les contributions ici réunies, sur les travaux d'Yves Jeanneret, pour décrire précisément le contenu des supports convoqués et mettre en évidence leur fonction. 
Par exemple, A. Cattelani et A. Errecart montrent comment l'hétérogénéité sémiotique des rapports RSE est organisée et mise au service de l'argumentation et d'un récit des entreprises. En particulier, les photographies de parties prenantes, qui accompagnent le texte, concourent à humaniser l'acteur économique qu'est l'entreprise et viennent construire le récit et l'image d'une entreprise altruiste.

L’hétérogénéité sémiotique et textuelle qui caractérise le rapport est également étudiée en détail par 0 . Vallée. L'auteure montre la manière dont s'agencent différents procédés et différents types de discours (narratif, argumentatif, descriptif) qui «construisent une représentation des pauvres et une représentation d'une solution à cette pauvreté et les modalités pratiques, idéologiques et éthiques de sa mise en œuvre», et qui sont ainsi «mobilisés pour construire, cadrer et justifier un savoir partagé sur le microcrédit». Parallèlement à des formes textuelles garantissant un «effet de scientificité », comme les listes de chiffres et les diagrammes, ces documents convoquent des formes plus subjectivisées comme le récit de vie, le témoignage, toutes concourant à une représentation positive du microcrédit. On voit avec ces études que la catégorie «description » ne suffit pas pour rendre compte de la visée informative, qu'il faut aussi convoquer les notions de récit et de narration, notions sur lesquelles la problématique des effets du rapport va nous amener à revenir.

\section{Carrière et efficience des rapports : quelle performativité?}

En s'intéressant au genre du rapport, on s'expose à la classique question des " effets » : rapports d'entreprise ou rapports d'activité ne sont-ils pas des textes entièrement routinisés et convenus, auxquels leurs destinataires mêmes ne prêteraient qu'une attention distraite? S'agissant des rapports publics, ne finissent-ils pas bien souvent dans les armoires - pour ne pas dire dans les poubelles - des ministères? Et pour quelques mesures importées de leurs suggestions 5 , pour quelques lois dès longtemps préméditées mais commodément imputées au texte remis à un ministre ${ }^{6}$, combien de missions inutiles et combien de rapports restés lettre morte? Le genre bien sûr n'a pas force de loi, et sa dimension normative même semble parfois mal assurée : on se souvient

5. Nous n'en prendrons pour exemple que la création de l'Agence du patrimoine immatériel de l'État, et autres mesures préfigurées par le Rapport Jouyet-Lévy surl' "économie de l’immatériel » (2006), texte dont les enjeux idéologiques ont été analysés en détail par Pierre Musso (2007).

6. On citera ici la loi El Khomri, directement issue de propositions du Rapport Badinter sur les «principes essentiels du droit du travail» (2016), mais aussi adossée à de plus anciennes recommandations du MEDEF. Voir à ce sujet l'article publié dans Le Monde du 19 février 2016 par Pierre Breteau et Samuel Laurent, «Droit du travail : une réforme directement inspirée des propositions du Medef et de la Droite » 〈http://www.lemonde.fr/les-decodeurs/article/2016/02/19/ droit-du-travail-une-reforme-directement-inspiree-des-propositions-du-medef-et-de-ladroite_4868716_4355770.html> (consulté le 3 avril 2017). 
du rapport Bénistiz bien vite escamoté, à peine l'encre séchée, victime de sa propre indigence et de son évidente stupidité. D’autres, pour être moins caricaturaux, n'en font pas moins pâle figure.

Si pourtant le rapport peut entrer dans la catégorie des discours d'autorité (Monte et Oger, 2015; Oger, 2013), c'est d'abord au sens où ses rédacteurs, membres par exemple de commissions et instances d'évaluation ou encore professionnels spécialisés, apparaissent comme des locuteurs autorisés, mandatés et adoubés par des institutions nationales ou internationales (Chevallier, 1996; Eymeri-Douzans, 2010), ou encore par de grandes organisations publiques ou privées (Hurez, 2008) ${ }^{8}$. C'est aussi en tant qu'il prétend fournir des éléments techniques ou «factuels », narratifs ou réflexifs au service d'une instance de décision ou d'évaluation (cf. supra) : en cela il soutient les mécanismes par lesquels se construit et se consolide l'autorité des décisions prises sur la foi de ses conclusions ou assertions.

Il alimente notamment les savoirs opératoires sur lesquels prétendent se fonder la décision et l'action raisonnées : principal outil de légitimation dans les «institutions hégémoniques», l'expertise - et plus généralement la connaissance - apparaît comme un recours envahissant pour justifier les politiques, au détriment - notamment - de savoirs réputés «locaux» et de la délibération collective (Della Faille et al., 2016). Si l'on demeure dans le cadre des systèmes démocratiques - excluant par là le cas des discours autoritaires / dictatoriaux - et si l'on met de côté le cas particulier des discours réglementaires et juridiques - auxquels s'attache une forme spécifique de performativité, la relation complexe qu'entretiennent discours autorisés et performativité nous semble rencontrer deux grands axes de réflexion.

Le premier est fourni par les sciences du langage et plus largement par les disciplines du discours, et souligne l'écart entre dimension illocutoire et dimension perlocutoire des énoncés (Austin, 1970; Kerbrat-Orecchioni, 2001), entre l'acte de langage et son résultat effectif, ou encore entre les places - inscrites en discours et ménagées dans les énoncés - et les positions - occupées ou modifiées dans l'espace social (Flahault, 1978).

L'incidence de discours institutionnalisés dans le secteur public comme dans les grandes organisations incite également à se tournervers une série de travaux menés en sociologie et plus particulièrement vers la manière dont la notion de performativité a été retravaillée dans le sillage des recherches de Michel Callon?.

7. Sur la prévention de la délinquance (2004), rapport préliminaire de la commission Prévention du groupe d'études parlementaire sur la sécurité intérieure, présidée par Jacques-Alain Bénisti. La version initiale de ce rapport, qui a fait ensuite l'objet de quelques palinodies, peut être lue ici : $\langle$ http://www.afrik.com/IMG/pdf/rapport_BENISTI_prevention.pdf〉 (consulté le 3 avril 2017).

8. La remise des rapports constitue d'ailleurs un rituel ordinaire de l'activité politicoadministrative, fournissant les conditions routinisées de leur médiatisation.

9. Callon, 1998 b et 2009 : ces deux articles reviennent sur la référence fondatrice, Callon, 1998 a. Voir aussi Denis et al., 2006. 
Donald MacKenzie (2006) a proposé à cet égard une contribution décisive et particulièrement claire, en montrant la capacité d'un modèle, ou d'une procédure, non seulement à s'inscrire dans les pratiques des acteurs mais aussi à rendre la réalité plus proche de la prévision, à la rendre pour ainsi dire plus conforme à la théorie.

Cette capacité d'agir ne doit pas pour autant être réduite à l'efficacité d'une prophétie auto-réalisatrice ${ }^{10}$ : loin d'opérer par le simple travail de l'idéologie qui amènerait les acteurs à rendre le monde conforme à leurs représentations, elle passe par le truchement de dispositifs qui viennent équiper les acteurs, et qui disposent d'une opérativité propre. C'est sans doute ce sens donné à « performativité » qui permet le mieux d'éclairer la nature des « effets » produits par nombre de rapports publics ou d'entreprises : en proposant des catégories supposément « descriptives», des comptages et chiffrages (Bouchard, 2008), des désignations tout autant que des préconisations, les rapports contribuent à la construction de référentiels et pourvoient les acteurs - notamment les acteurs publics - en «équipements experts» (Trépos, 1996, p. 90).

Schèmes de pensée ou de raisonnement, traditions étatiques, normes juridiques ou techniques, labels nationaux ou européens, et plus généralement formes instituées de modélisation contribuent sous cette appellation générale à la standardisation des procédures et des conclusions des discours d'experts, alimentant un mouvement de mise en ordre, fixé par des repères préétablis. De tels outils d'aide à la décision, méthodes pour réduire les incertitudes inhérentes à l'action politique ou économique, procédures d'objectivation des « réalités sociales » remplissent bien leur fonction de discours d'experts, au service de la conduite de l'action. S'agissant des rapports publics, de tels équipements entrent dans la composition des « instruments de l'action publique » décrits par la science politique (Lascoumes, 2007; Lascoumes et Le Galès, 2004), abritant des conceptions politiques dans les plis de leurs procédures objectivantes.

Le récit lui-même des événements relatés dans les rapports peut prendre une dimension monologique ou dogmatique qui tend à imposer une version vraisemblable et une interprétation qui font autorité. On peut en prendre pour exemple le Rapport Cullen, rapport d'enquête publique britannique relatif à l'accident industriel survenu en 1988 sur une plateforme pétrolière offshore, faisant près de 200 morts. Pour Andrew Brown (2003), la relation des faits mêmes engage des régimes de vérité qui fournissent les prémisses et sous-tendent les raisonnements mis en œuvre dans la prise de décision : la hiérarchisation et la présentation des témoignages, l’interprétation des données techniques servent et accréditent une version autorisée de l'événement qui, sans être nécessairement ni fausse ni mensongère, fait silence

10. Callon, 2009. D. Mackenzie (2006, p. 44-45) ajoute que l'adoption du modèle étudié ne peut être réduite à l'autorité dont peuvent jouir le discours économique en général ou tel modèle en particulier, et met au jour d'autres raisons pratiques, liées notamment à la coordination des acteurs professionnels et à la recherche de compétitivité. 
sur les modalités de sa propre construction et sur les choix opérés par les rapporteurs.

Ce rôle dévolu à la narration se retrouve sous une autre forme dans les micro-récits, constituant autant de témoignages des «bonnes pratiques », qui ont envahi les discours des institutions et singulièrement les rapports publics : la description d'un monde meilleur, à portée de la bonne volonté de tout un chacun, y côtoie les mises en garde explicites et formes de mobilisation très directes des acteurs. Elle fournit la caution d'un discours normatif supposé orienter très directement les politiques publiques ${ }^{11}$. L'article d'Odile Vallée, cité plus haut, montre ici-même comment récits et portraits viennent se combiner à l'argumentation par les chiffres dans les rapports d'État de la Campagne du Sommet du microcrédit : ils y construisent conjointement la dimension normative des rapports, célébrant la puissance transformatrice de la Campagne. En cela l'autorité des récits, comme celle des chiffres, se soutient d'une prétention à reconfigurer le monde.

D'autres rapports tendent plus directement encore à stabiliser les contours et le cadrage d'une doctrine d'action publique ${ }^{12}$ : Philip Schlesinger (2007 et 2009) a montré comment les discours d'experts britanniques sur les industries créatives, repris dans un certain nombre de rapports, se sont consolidés en une véritable doctrine qui a servi de référence incontestée et de socle à l'action des pouvoirs publics. Et l'on trouvera une autre illustration de ce processus de stabilisation dans les travaux d'Éric Dagiral (2007) sur la contribution d'une collection de rapports publics à la construction en France d'une «administration électronique». En cela les rapports participent pleinement et de manière décisive au processus de «cadrage », largement décrit et analysé dans les travaux sur la construction des problèmes publics, et indissociable de leur «carrière »(Neveu 2015, chap.3).

L'ensemble de ces observations permet d'envisager le rapport comme un genre qui opère une médiation singulière entre le compte rendu (voir supra) et les textes plus directement prescriptifs qui encadrent et accompagnent la conduite de l'action par les dirigeants. C'est sans doute dans l'article de L. Renard et N. Truan que l'on trouvera l'illustration la plus détaillée d'un tel processus : se penchant sur un corpus de 29 rapports français et allemands portant sur la question de l'intégration, elles en examinent les «incipit», qui concentrent les considérations stratégiques, et montrent comment les rapports se présentent eux-mêmes comme "cadreurs du débat», parfois susceptibles d'apaiser une société en proie à l'émotion, ou de s'opposer aux approches réputées simplificatrices. Dans le même temps, injonctions et interpellations

11. Levoin et Oger, 2012. Dans cet article sont notamment commentés trois rapports publics relatifs aux politiques de promotion de la «créativité».

12. Pour une lecture de la notion de cadrage (empruntée à Erving Goffman), envisagée dans ses relations avec les problématiques ici évoquées, voir Callon, $1998 \mathrm{~b}$. 
tentent de mobiliser les destinataires et d'infléchir les politiques d'intégration préconisées. Au service de cette visée, les textes s'efforcent de forger des catégories réfléchissant sur des désignations comme «migrants » ou « étrangers », d'élaborer des catégories permettant la production de statistiques («population à arrière-plan migratoire » ou « issue de l'immigration »). Or les analogies observées entre les deux sous-corpus français et allemands - en dépit des différences entre les contextes politiques et institutionnels dans les deux pays pourraient s'expliquer précisément par les contraintes d'un genre discursif fondé sur cette double injonction à informer l'opinion publique mais aussi à infléchir les politiques publiques.

Enfin, la pratique même de la rédaction des rapports a des effets en retour sur les mondes sociaux considérés et sur les normes et les usages qui y sont prescrits, comme le montre la note rédigée par Anne Piponnier : ainsi du monde universitaire, où le rapport de recherche, rédigé dans le contexte de contrats de recherches sur financements publics ou privés, et supposé justifier de l'avancée des travaux, tend à être considéré et comptabilisé comme publication scientifique à part entière. Discours de commande étroitement contraint par un «formulaire» qui en fournit la trame et enserre les observations dans une séquence standardisée, il réinvestit et transforme à la fois les usages académiques, opérant des formes de rationalisation dans le compte rendu de l'activité de projet. Et il infléchit d'une autre manière encore les pratiques de recherche en tant qu'il entre dans les stratégies de communication et de promotion de la recherche par les équipes ou les porteurs de projet : souvent peu accessible mais largement référencé, il opère comme un objet de médiation entre recherche et expertise, et transforme en profondeur les normes de l'écriture scientifique et par là l'activité même de recherche qu'il soumet à un impératif institutionnel d'accountability.

L'ensemble des articles réunis dans ce dossier illustre la place singulière que jouent la production et la circulation des rapports dans différentes sphères d'activité. Dans leur double prétention à décrire et à prescrire, ils remplissent en somme une double fonction similaire à celle qu'Alain Desrosières (2008, p.187-203) assigne aux statistiques : refléter et instituer le monde social.

\section{Références}

ADAM Jean-Michel, 2011, Genres de récits : narrativité et généricité des textes, Louvainla-Neuve, L'Harmattan-Academia.

Austin John L., 1970, Quand dire, c'est faire, Paris, Éd. du Seuil [Titre original: How to Do Things with Words, Oxford, Oxford University Press, 1962].

Bacot Paul, Desmarchelier Dominique, Rémi-GIraud Sylvianne éd., 2012, «Chiffres et nombres dans l'argumentation politique », Mots. Les langages du politique, n 100. 
BAKHTINe Mikhaïl, 1984, Esthétique de la création verbale, Paris, Gallimard.

BOUCHARD Julie éd., 2008, "La communication nombre», MEI. Médiation et information. Revue internationale de communication, $\mathrm{n}^{\circ} 28$.

BRANCA-Rosoff Sonia, 1999a, «Types, modes et genres entre langue et discours », Langage et société, n०87, p.5-24.

BRAnCA-Rosoff Sonia, 1999b, "Des innovations et des fonctionnements de langue rapportés à des genres », Langage et société, n87, p. 115-129.

Brown Andrew D., 2003, "Authoritative Sensemaking in a Public Inquiry Report», Organization Studies, vol. 25, nº 1, p. 95-112.

CALLON Michel éd., 1998a, The Laws of Markets, Oxford, Blackwell.

CALLon Michel, 1998b, «An Essay on Framing and Overflowing: Economic Externalities Revisited by Sociology», The Sociological Review, vol.46, nº spécial («Sociological Review Monograph Series : The Laws of the Markets »), p. 244-269.

CALLON Michel, 2009, "Elaborating the Notion of Performativity», Le Libellio d'Aegis, vol.5, no1, p.18-29; disponible sur Internet : 〈https://hal-mines-paristech. archives-ouvertes.fr/hal-00460877> (consulté le 3 avril 2017).

CASSIN Barbara, 2014, Derrière les grilles : Sortons du tout-évaluation, Paris, Fayard/ Mille-et-une nuits.

Chevallier Jacques, 1996, «L'entrée en expertise», Politix, n³ 36, p. 33-50.

Cussó Roser, GobIN Corinne, 2008, «Du discours politique au discours expert : le changement politique mis hors débat?», Mots. Les langages du politique, n88, p. 5-11.

DAGIRAL Eric, 2007, "La construction de l'administration électronique au prisme des rapports publics», Terminal, 2007, nº 99-100, p. 33-44.

Della Faille Dimitri, La France-Moreau Valérie et Paradis-Charette Laurent, 2016 «Discours à propos du rôle de l'expertise dans les processus de prise de décision en développement international », Politique et sociétés, vol. 35, n²-3, p. 215-237.

DENIS Jérôme, 2006, "Les nouveaux visages de la performativité », Études de communication, $\mathrm{n}^{\circ} 29$, p. 8-24.

Desrosières Alain, 2008, L'argument statistique. I. Pour une sociologie historique de la quantification, Paris, Presses de l'École des Mines.

EymerI-DouzAns Jean-Michel, 2010, "Ce que faire l'expert pour la Commission européenne veut dire. Essai d'auto-analyse d'une trajectoire de socialisation», La fabrique des «Européens». Processus de socialisation et construction européenne, H. Michel et C. Robert éd., Strasbourg, Presses universitaires de Strasbourg, p. 287-312.

Flahault François, 1978, La parole intermédiaire, Paris, Éd. du Seuil.

HUREz Stéphanie, 2008, «Des statistiques à mi-chemin entre dits et non-dits : étude des rapports d'activités disponibles sur le secteur du téléachat», MEI. Médiation et information. Revue internationale de communication, $\mathrm{n}^{\circ} 28, \mathrm{p} .75-91$.

JALENQUES Pierre, 2002, «Étude sémantique du préfixe $R E$ en français contemporain : à propos de plusieurs débats actuels en morphologie dérivationnelle», Langue française, $\mathrm{n}^{0} 133, \mathrm{p} .74-90$.

Kerbrat-Orecchioni Catherine, 2001, Les actes de langage dans le discours, Paris, Nathan. 
LASCOUMES Pierre, 2007, "Les instruments d'action publique, traceurs de changement : l'exemple des transformations de la politique française de lutte contre la pollution atmosphérique (1961-2006) », Politique et sociétés, vol. 26, n²-3, p.73-89.

LAscoumes Pierre, Le Galès Patrick éd., 2004, Gouverner par les instruments, Paris, Presses de Sciences Po.

Levoln Xavier, Oger Claire, 2012, "Concours de créativité dans l'enseignement : approche critique des politiques publiques d'innovation », Communication et langages, 2012, vol.173, p. 113-128.

MackenzIE Donald, 2006, "Is Economics Performative? Option Theory and the Construction of Derivatives", Journal of the History of Economic Thought, vol. 28, no 1 , p. 29-55.

MaINGUENEAU Dominique, 2002, "Les rapports des organisations internationales: un discours constituant?», Les mots du pouvoir. Sens et non-sens de la rhétorique internationale, G. Rist éd., Paris, PUF; Genève, Institut universitaire d'études du développement, p.119-132; disponible sur Internet 〈http://books.openedition. org/iheid/2443> (consulté le 3 avril 2017).

Maingueneau Dominique, 2004, Le discours littéraire : paratopie et scène d'énonciation, Paris, A. Colin.

Mellet Caroline, Sitrı Frédérique, 2010, «Nom de genre et institutionnalisation d'une pratique discursive : les cas du signalement d'enfant en danger et de l'interpellation parlementaire», Actes du $2^{e}$ Congrès mondial de linguistique française : La Nouvelle-Orléans, 12-15 juillet 2010, F. Neveu et alii éd., p.781-795; disponible sur Internet : 〈http://www.linguistiquefrancaise.org/articles/cmlf/pdf/2010/01/ cmlf2010_000175.pdf> (consulté le 7 avril 2017).

MONTE Michèle, Oger Claire éd., 2015, "Discours d'autorité : des discours sans éclats?", Mots. Les langage du politique, nº107.

Musso Pierre, 2007, «Une critique de l'“économie de l'immatériel” vue par le rapport Jouyet-Lévy», Quaderni. Communication, technologies, pouvoir, nº64, p. 81-88.

NÉE Émilie, SITRI Frédérique, Fleury Serge, 2014, "L'annotation du pronom “nous" dans un corpus de rapports éducatifs. Objectifs, méthodes, résultats», communication aux $12^{\text {es }}$ Journées internationales d'Analyse statistique des données textuelles (JADT), 〈http://lexicometrica.univ-paris3.fr/jadt/jadt2014/01-ACTES/41JADT2014.pdf> (consulté le 3 avril 2017).

NÉE Émilie, SITRI Frédérique, VenIARD Marie, 2014, «Pour une approche des routines discursives dans les écrits professionnels", Actes $d u 4^{e}$ Congrès mondial de linguistique française : Berlin, 19-23 Juillet 2014, F. Neveu et alii éd., Les Ulis, ESPD Sciences, vol. 8, p. 2113-2124; disponible sur Internet : 〈http://dx.doi.org/10.1051/ shsconf/20140801195> (consulté le 3 avril 2017).

NÉE Émilie, SITRI Frédérique, VENIARD Marie, 2016, "Les routines, une catégorie discursive pour caractériser les genres?», Lidil, nº53 ("Phraséologie et genres de discours»), p. 71-93.

NÉe Emilie, Sitrı Frédérique, Veniard Marie, Fleury Serge (à paraître), «Routines discursives et séquentialité dans des écrits professionnels : la mise au jour d'une 
séquence évaluative?», Corpus, nº 15 ("Segments et séquences textuelles : méthodologie et caractérisation »).

NeVEu Erik, 2015, Sociologie politique des problèmes publics, Paris, A. Colin.

OGER Claire, 2013, "L'institution du Neutre», Discours d'autorité, discours autorisés. Faire référence et dire l'institution, Dossier d'Habilitation à diriger des recherches en Sciences de l'information et de la communication, Université Paris-Sorbonne, CELSA.

SCHLESINGER Philip, 2007, "Creativity: from Discourse to Doctrine?», Screen, vol.48, $\mathrm{n}^{\circ} 3$, p. 377-387.

SCHLESINGER Philip, 2009, "Creativity and the Experts. New Labour, Think Tanks, and the Policy Process », The International Journal of Press/Politics, n ${ }^{14}$, p. 3-20.

SITRI Frédérique, 2015, «Le genre comme principe de détermination du discours ", Parcours en analyse du discours : enjeux et méthode. Autour d'écrits professionnels, Synthèse du Dossier d'Habilitation à diriger des recherches en Sciences du langage, Université Sorbonne nouvelle-Paris 3, p. 91-246.

Todorov Tzvetan, 1981, Mikhaïl Bakhtine : le principe dialogique suivi de Écrits $d u$ Cercle de Bakhtine [textes choisis de V. N. Volochinov et Mikhail Bakhtine]. Paris : Éd. du Seuil.

TRÉPos Jean-Yves, 1996, La sociologie de l'expertise, Paris, PUF.

VogüE Sarah de, 1999, "Construction d’une valeur référentielle : entités, qualités, figures", Travaux linguistiques du Cerlico [Cercle linguistique du centre et de l'ouest], n' ${ }^{0} 12$, p. 77-106. 